\title{
Significance of Non-phase Locked Oscillatory Brain Activity in Response to Noxious Stimuli
}

\author{
Raphaël Dufort Rouleau, Lydia Lagrandeur, Kathya Daigle, Dominique Lorrain, \\ Guillaume Léonard, Kevin Whittingstall, Philippe Goffaux
}

\begin{abstract}
Background: Although current pain-evoked electroencephalographic (EEG) studies provide valuable information regarding human brain regions involved in pain, they have mostly considered neuronal responses which oscillate in phase following a painful event. In many instances, cortical neurons respond by generating bursts of activity that are slightly out of phase from trial-to-trial. These types of activity bursts are known as induced brain responses. The significance of induced brain responses to pain is still unknown. Methods: In this study, 23 healthy subjects were given both non-painful and painful transcutaneous electrical stimulations in separate testing blocks (stimulation strength was kept constant within blocks). Subjective intensity was rated using a numerical rating scale, while cerebral activity tied to each stimulation was measured using EEG recordings. Induced brain responses were identified using a time frequency wavelet transform applied to average-removed single trials. Results: Results showed a pain-specific burst of induced theta activity occurring between 180 and $500 \mathrm{~ms}$ post-shock onset. Source current density estimations located this activity within the dorsolateral prefrontal cortex (DLPFC, bilaterally), however, only right DLPFC activity predicted a decrease in subjective pain as testing progressed. Conclusion: This finding suggests that non-phase locked neuronal responses in the right DLPFC contribute to the endogenous attenuation of pain through time. Perspective: This article presents neuroimaging findings demonstrating that, in response to pain, non-phase locked bursts of theta activity located in the right dorsolateral prefrontal cortex are associated with a progressive decrease in subjective pain intensity, which has potentially important implications regarding how humans endogenously control their experiences of pain.
\end{abstract}

RÉSUMÉ: Signification de l'activité cérébrale induite en réponse aux stimuli douloureux. Contexte: Bien que les études électroencéphalographiques concernant la réponse à la douleur fournissent des informations précieuses sur les régions du cerveau humain impliquées dans la perception de la douleur, elles portent surtout sur les réponses neuronales qui oscillent en phase après un événement douloureux. Dans plusieurs cas, les neurones corticaux répondent en générant de l'activité qui est légèrement déphasée d'une fois à l'autre. Ce type d'activité est appelé réponse cérébrale induite. La signification des réponses cérébrales induites par un évènement douloureux demeure inconnue. Méthode: Cette étude porte sur 23 sujets sains soumis à des blocs séparés de stimulation électrique transcutanée non douloureuse et douloureuse. La force de la stimulation demeurait constante à l'intérieur de chacun des blocs de stimulation. Une échelle d'évaluation numérique a été utilisée pour estimer l'intensité de la stimulation ressentie par le sujet pendant que l'activité cérébrale liée à chaque stimulation était mesurée par enregistrement ÉEG. Les réponses cérébrales induites ont été identifiées au moyen d'une transformation en ondelettes tempsfréquence appliquée à des épreuves uniques avec soustraction de la réponse évoquée moyenne de la réponse obtenue. Résultats: Les observations ont mis en évidence une augmentation d'activité thêta spécifique à la douleur qui survient entre 180 et $500 \mathrm{~ms}$ après le début de la stimulation. Les estimations de la densité du courant source ont permis de localiser cette activité dans le cortex préfrontal dorso-latéral (CPFDL) bilatéralement. Cependant, seulement l'activité du CPFDL droit prédisait une diminution de la douleur subjective à mesure que le testing progressait. Conclusion: Cette observation suggère que les réponses ÉEG induites et provenant du CPFDL droit contribuent à l'atténuation endogène de la douleur avec le temps. Perspective: Cet article démontre qu'en réponse à la douleur, une augmentation d'activité thêta, déphasée temporellement et localisée dans le CPFDL droit est associée à une diminution progressive de l'intensité de la douleur subjective, ce qui pourrait avoir des implications importantes en ce qui a trait à la façon dont les humains contrôlent leur expérience de la douleur.

Keywords: Dorsolateral prefrontal cortex, EEG, Induced brain activity, PAIN, Theta

doi:10.1017/cjn.2015.294

Can J Neurol Sci. 2015; 42: 436-443

Nociceptive processing in the human central nervous system can be recorded in different ways, including through the use of hemodynamic and neuroelectric methods. ${ }^{1-4}$ Recently, high temporal resolution electrophysiological recordings have become

From the School of Rehabilitation (RDR,LL,KD,GL,PG); Department of Diagnostic Radiology (KW); Sherbrooke Molecular Imaging Center, Department of Nuclear Medicine and

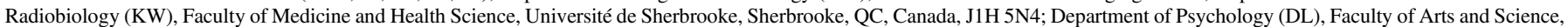
Université de Sherbrooke, Sherbrooke, QC, Canada, J1K 2R1.

Received November 4, 2014. Final Revisions Submitted April 21, 2015.

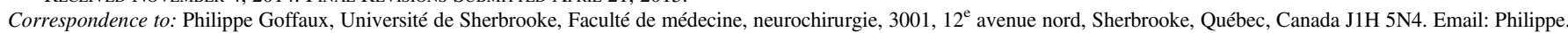
Goffaux@USherbrooke.ca 
popular as a way of better understanding when painful afferent volleys to the cortex are processed and how these relate to the psychophysical properties of experienced pain. ${ }^{5}$ Human electroencephalographic (EEG) recording studies together with source localization analysis of generators have: i) confirmed the presence and sub-second activation pattern of brain structures tied to pain intensity processing, ${ }^{6}$ ii) shown that individual differences in pain sensitivity partly depend on individual differences in the early activation response of the precuneus, ${ }^{7}$ iii) demonstrated that the primary somatosensory cortex, when activated soon after a painful stimulus, does indeed participate in the processing of painful experiences, ${ }^{8}$ and, iv) shown that regulatory areas of the orbitofrontal cortex and amygdala participate in the initial activation and control of descending bulbo-spinal pain inhibition circuits. ${ }^{9}$

Although these studies are useful in their ability to highlight how the human brain responds to painful experiences, they have only considered neuronal networks which oscillate in phase to painful afferent volleys. This type of activity known as evoked or phase locked - can be instructive regarding the role of synchronization in post-stimulus EEG amplitude changes (i.e., post-stimulus reorganization of oscillatory neuronal networks). However part of the brain's response to an afferent sensory stimulus is not rigidly phase locked to stimulus onset but, instead, will vary slightly from trial-to-trial. Bursts of such asynchronous oscillatory activity are called induced - or non-phase locked responses. These have very rarely been explored in pain research. Evoked and induced brain responses are thought to represent different neurophysiological brain processes. Evoked responses, for example, are thought to reflect the resonant activity of cortical neurons in response to incoming sensory information. ${ }^{10}$ Induced responses, on the other hand, are thought to reflect the indirect synchronous firing of neuronal assemblies produced by large fluctuations in the membrane potential of neurons. ${ }^{11}$ These fluctuations occur close to, and depend on, the coherent activation patterns normally triggered by incoming sensory information.

Few studies have investigated induced EEG response in pain. The first is a study by Mouraux et al., ${ }^{12}$ which tested for the possible interaction between $\mathrm{A} \delta$ and $\mathrm{C}$ fiber-evoked EEG signatures to pain. Using both phase and non-phase locked measurements of $\mathrm{A} \delta$ and $\mathrm{C}$ fiber-mediated responses to pain (uncoupled using laser stimuli and reaction time tasks), the authors showed that $\mathrm{A} \delta$ and $\mathrm{C}$ fibers produce the same brain response, which, when already engaged by rapidly-travelling $A \delta$ fibers, do not need to be reactivated by slowly-travelling $\mathrm{C}$ fibers. The second key paper was published by Babiloni et al. ${ }^{13}$ and showed that EEG oscillatory responses (both phase and non-phase locked) to a painful stimulation occur in both hemispheres. Although the EEG response recorded by Babiloni et al. ${ }^{13}$ was dominated by phase locked activity, a significant non-phase locked burst of alpha activity was observed. More importantly, it was shown that, depending on the frequency of interest, phase and non-phase locked EEG oscillations have different scalp topographies and, thus, could be related to different underlying sources and involve different pain processing functions. Despite being important to the field of pain neuroimaging, these two papers did not seek to address the functional significance of non-phase locked EEG responses to pain. To address this issue, we analysed EEG responses to pain in the time-frequency domain, source-localized underlying brain generators and explored how these changes relate to the psychophysical properties of pain.

\section{Methods}

\section{Subjects}

Thirteen women (mean age \pm standard error, $23.8 \pm 0.65$ years) and ten men (mean age \pm standard error, $26.3 \pm 2.03$ years) participated in the experiment. The study was carefully explained to all participants and all provided written, informed consent. The study was approved by the research ethics committee of the Centre Hospitalier Universitaire de Sherbrooke (CHUS).

\section{Subjective ratings}

Verbal numerical rating scales (NRS) were used to evaluate the intensity of all somesthetic sensations. An innocuous NRS was used to evaluate non-painful sensations and ranged from 0-100, where 0 was defined as "no sensation" and 100 was defined as "extremely intense, but not painful". A noxious NRS was used to evaluate painful sensations and also ranged from $0-100$, but this time, 0 was defined as "no pain" and 100 was defined as "intolerable pain intensity". In order to distinguish between the scales, participants had to precede all innocuous evaluations by the word "non-painful". Using separate 0-100 scales to assess non-painful and painful sensations respectively was preferred to the use of a single 0-200 scale (where 100 would have represented the pain perception threshold) because it was feared that a single 0-200 scale might have been less intuitively used by participants, and thus, might have led to important evaluation errors.

\section{Sural nerve stimulations}

Both non-painful and painful sensations were provoked using transcutaneous electrical stimulations of the right sural nerve. The sural nerve was stimulated over its retromalleolar path. Stimulations consisted of a volley of 10 electrical pulses (square waves $1 \mathrm{~ms}$ pulse duration) administered at a rate of $320 \mathrm{~Hz}$ using a constant current stimulator. A stimulation volley lasted $31 \mathrm{~ms}$. Stimulations were provided using a pseudorandom inter stimulus interval of 6-12 s (geometrical distribution with a mean of 7.4 stimuli per minute). The use of a jittered design allowed us to control for pain-expectation effects, which are known to influence subjective pain reports ${ }^{14}$. Stimulations were provided in two separate testing blocks. Each block lasted 4.5 minutes with an inter-block interval of 5 minutes. The first block contained 33 stimuli provided at a stimulation intensity level necessary to provoke strong tactile (but non-painful) sensations and corresponding to a score of 50 on the innocuous NRS (individually tailored). The second testing block also contained 33 stimuli but this time the intensity level was adjusted to ensure the subjective experience of mild pain - corresponding to a score of 15 on the noxious NRS. Mild, as opposed to moderate or elevated pain was targeted in our study because sural nerve sensations are typically experienced as more unpleasant than intense by participants. As a result, high levels of targeted pain intensity run the risk of causing extremely high levels of pain unpleasantness and thus elevated drop-out rates. Stimulation intensity always remained constant within blocks. The non-painful testing block was always presented before the painful testing block. This is important because painful testing blocks can potentially produce spinal sensitizing effects that can carry-over and affect the evaluation of all subsequent blocks. The use of an incremental testing design, 
therefore, prevented potential carry-over effects from the painful to the non-painful block.

\section{Somatosensory-evoked brain potentials}

Shock-evoked somatosensory-evoked potentials (SEPs) were recorded from 32 electroencephalographic electrodes arranged on the scalp in a grid corresponding to the international 10-20 system (32-electrode cap). Using a Brain Vision recording system (Brain Products GmBh, Munich, Germany), our signal was sampled at $250 \mathrm{~Hz}$ and bandpass filtered between 0.5 to $30 \mathrm{~Hz}$. A common average reference montage was used. Impedance at each electrode position was kept below $5 \mathrm{k} \Omega$. Trials contaminated by eye-blinks and movements were corrected using an independent component analysis (ICA) algorithm (Brain Vision Analyzer software, Brain Products $\mathrm{GmbH}$, Munich, Germany). After ICA, an additional baseline correction $(-100$ to $0 \mathrm{~ms}$, where $0 \mathrm{~ms}$ represents shock onset) was applied. Each trial spanned a $-100 \mathrm{~ms}$ to $600 \mathrm{~ms}$ time period.

\section{Time-frequency analysis}

Time-frequency (TF) analysis was run using a continuous Morlet wavelet transform (CMT). The CMT was computed using absolute values of the raw EEG data and was baseline corrected. Our CMT baseline correction method was performed by calculating the average value of the wavelet coefficients between $-100 \mathrm{~ms}$ to $0 \mathrm{~ms}$ and subtracting this value from all wavelet coefficients within the entire epoch (i.e., $-100 \mathrm{~ms}$ to $600 \mathrm{~ms}$ ) and for all corresponding frequency levels $(1 \mathrm{~Hz}$ resolution). A subtraction approach to baseline correction was preferred to a percentage approach (which calculates a percentage change value from baseline) because the subtraction approach minimizes overestimations in synchronized brain responses. ${ }^{15}$ Following baseline correction, we normalized our wavelet functions using a relative wavelet power method, which ensured that our wavelet function would have the same energy across all scales. This approach produces wavelet power spectra that are directly comparable to each other across all scales. Time-frequency analyses were applied only to average-removed single trials, which isolated the induced (i.e., non-phase locked) component of the SEP response. ${ }^{16}$ Time-frequency analyses were conducted separately on all trials within painful and non-painful blocks.

\section{Cortical mapping}

Visual inspection of the time frequency oscillation amplitude maps depicted in Figure 1 clearly show a pain minus non-pain burst of delta/theta activity spanning a 180 to $500 \mathrm{~ms}$ time period and located across frontal and posterior leads (Note: the difference in delta/theta scalp activity between pain and non-pain trials was statistically significant; see Results section below). To properly investigate the underlying source of this non-phase locked activity, we first segmented the pain and non-pain raw EEG data between 180 and $500 \mathrm{~ms}$. The segmented data were individually transformed into a cross-spectrum file with a discrete frequency range extending between 1 and $30 \mathrm{~Hz}$ and a frequency resolution of $1.95 \mathrm{~Hz}$ (this was the upper limit of our resolution given our segment length of $320 \mathrm{~ms}$ [i.e., $180 \mathrm{~ms}$ to $500 \mathrm{~ms}$ ]). Following the computation of both pain and non-pain cross spectrum files (completed separately for every participant), a source file was

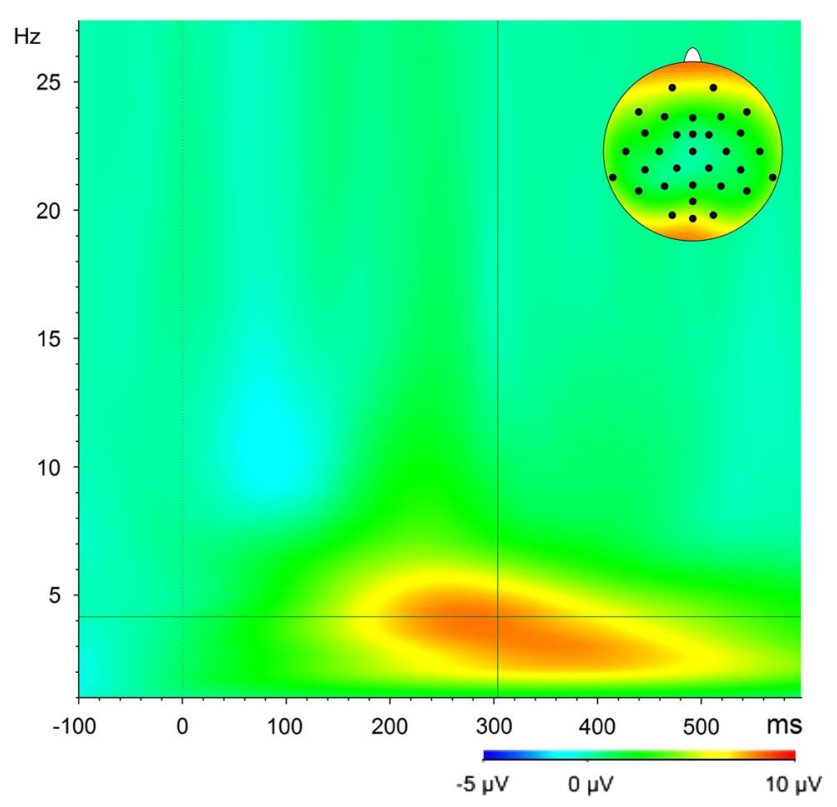

Figure 1: Time-frequency decomposition of non-phase locked oscillatory activity recorded at electrode $\mathrm{Oz}$ (distribution across the entire scalp is shown in upper right corner). Time frequency data shown here represents the activity difference between pain and nonpain trials. Estimations were obtained using a continuous Morlet transform applied to average-removed single trials. An event-related synchronization response is clearly visible between 180 and $500 \mathrm{~ms}$ and ranging between 1.5 and $7 \mathrm{~Hz}$.

computed to estimate the source generators at each discrete frequency ( 15 discrete frequencies in total). Non-phase locked data were transformed from scalp space to source space using standardized low-resolution electromagnetic tomography (sLORETA). Standardized low-resolution electromagnetic tomography is a method to estimate the localization of brain function by providing a solution to the inverse problem. ${ }^{17,18}$ It falls into the class of distributed source models that do not require a priori knowledge regarding the number of active sources and thus is advantageous for the localization of processes that depend on multiple activity distributions, as is the case for pain. ${ }^{1,19,20}$ The source space in sLORETA is calculated with the boundary element method applied to the standardized Montreal Neurological Institute (MNI) brain volume consisting of 6239 cortical gray matter voxels at five-mm resolution. In the present study, the computation of sLORETA (which provides electric current density values) was applied, for each participant, to the non-phase locked data obtained at each of our 15 discrete frequencies on both non-pain and pain trials.

\section{Experimental protocol}

Prior to testing, a pre-experimental session was carried out to individually determine the current intensities to be used during the testing session. Participants were asked to rate the strength of the sensation they felt following each practice stimulation during the pre-experimental session. This allowed us to determine the current intensity in milliamperes $(\mathrm{mA})$ required to elicit both non-painful (i.e., 50 on the innocuous NRS) and mildly painful (i.e., 15 on the noxious NRS) sensations for each participant. It also allowed us to 
determine the pain perception threshold (in $\mathrm{mA}$ ). During testing, intensity ratings were recorded three times during the non-painful block and three times during the painful block. For each block, intensity ratings were obtained once every 1.5 minutes and reflected the average pain felt since the beginning of a block (for the first assessment) or since the preceding assessment (for the last two assessments). Having three scores within a given block allowed us to test for the stability of ratings within blocks. It also allowed us to calculate the difference between the last and first score within a block, giving us an opportunity to explore interindividual variations in subjective rating as testing time progresses.

\section{Statistical analyses}

\section{Subjective intensity ratings and sural nerve stimulation strength}

To ensure that the average intensity rating within a given block corresponded to the intensity value that was targeted for that block, we conducted two separate one-sample $t$-tests (one each for the non-painful and painful testing block). A two-way repeated measures analysis of variance (ANOVA) (Block X Rating Occasion) was also conducted to explore possible changes in subjective rating within testing blocks (i.e., rating changes through time). Bonferroni corrections were applied where appropriate.

\section{Pain-related cortical activation maps associated with non-phase locked SEP responses}

Cortical activity tied specifically to pain induced responses were estimated by comparing the electric current density maps obtained from the non-phase locked data in the non-pain condition from the current density maps obtained from the nonphase locked data in the pain condition. This comparison was made at each of our 15 discrete frequencies. Statistical significance was assessed through nonparametric permutation tests using 5000 randomizations accounting for multiple comparisons (i.e., multiple anatomical sites and multiple frequency intervals). Significance was set to $\mathrm{p}<0.05$ (two-tailed tests were always used). Statistical non-parametric mapping provided 3D $t$-maps for all consecutive frequencies in the 180 to $500 \mathrm{~ms}$ postshock epoch.

\section{Correlation analysis}

To explore the functional significance of pain induced responses, we correlated various psychophysical pain indices (i.e., pain threshold, sub- and supra- pain-threshold scores, pain sensitivity scores and change in subjective experience as stimulations repeat through time) with the source activity (in amperes per square meter, $\left(\mathrm{A} / \mathrm{m}^{2}\right)$ ) of pain-induced brain responses. The painrelated brain regions chosen for these correlations were limited to those regions (spatial local maxima) which were significantly different when pain and non-pain induced responses were compared (see description of this analysis in the preceding section). The psychophysical pain indices chosen in the current study were the pain threshold (in $\mathrm{mA}$ ), the sub- and supra- pain-threshold (both in $\mathrm{mAs}$ ), the pain sensitivity index (change in sural nerve stimulation current between the non-painful and painful blocks), and the change in the subjective rating between the last and first score within a block (both non-painful and painful, each calculated separately). Bonferroni corrections were applied to limit the risk of type I error.

\section{RESULTS}

\section{Subjective ratings}

Average NRS ratings for the non-painful (mean $=53.40 \pm$ standard error $(\mathrm{SE})=3.70$ ) and painful testing blocks (mean $=$ $15.10 \pm \mathrm{SE}=1.75$ ) were compared to their corresponding target NRS values (50 and 15, respectively). Numerical rating scale ratings for the non-painful testing block $\left(\mathrm{t}_{22}=0.92, \mathrm{p}=0.37\right)$ and for the painful testing block $\left(\mathrm{t}_{22}=0.058, \mathrm{p}=0.95\right)$ were not significantly different from their targeted values. For both testing blocks, intra-block subjective ratings (i.e., first, second, and third ratings) did not vary significantly from one another $\left(\mathrm{F}_{2,44}=2.81\right.$, $\mathrm{p}=0.07$; see Table 1).

\section{Source localization of non-phase locked SEP responses}

Visual inspection of the time frequency oscillation amplitude map depicting the difference between painful and non-painful stimulations clearly shows a burst of delta/theta activity spanning a 180 to $500 \mathrm{~ms}$ time period (See Figure 1) and localized across both frontal and posterior leads. This burst of localized activity resulted from significantly greater delta and theta power on pain trials than on non-pain trials at frontal leads (peak activity at electrode $F p 1, \mathrm{t}_{22}=2.55, \mathrm{p}=0.02$ ) and a marginal difference at posterior leads (peak activity at electrode $\mathrm{Oz} \mathrm{t}_{22}=1.74, \mathrm{p}=0.09$ ). Within the 2.9 to $4.9 \mathrm{~Hz}$ range, generators for this burst of induced, pain-related activity were localised to the left superior frontal gyrus (SFG; spatial local maximum at $\left.\mathrm{x}=-20, \mathrm{y}=30, \mathrm{z}=50 ; \mathrm{t}_{22}=3.75, \mathrm{p}<0.05\right)$ and right middle frontal gyrus (MFG; spatial local maximum at $\mathrm{x}=50, \mathrm{y}=15$, $\mathrm{z}=45 ; \mathrm{t}_{22}=3.83, \mathrm{p}<0.05$; see Figure $2 \mathrm{a}$ ). Within the 4.9 to $6.9 \mathrm{~Hz}$ range, generators were localized to the left (spatial local maximum at $\mathrm{x}=-25, \mathrm{y}=30, \mathrm{z}=55 ; \mathrm{t}_{22}=3.75, \mathrm{p}<0.05$ ) and right SFG (spatial local maximum at $x=25, y=25, z=55$; $\mathrm{t}_{22}=3.61, \mathrm{p}<0.05$ ), and, to the right MFG (spatial local maximum at $\mathrm{x}=45, \mathrm{y}=20, \mathrm{z}=40 ; \mathrm{t}_{22}=3.68, \mathrm{p}<0.05$; see Figure 2b).

\section{Correlation analyses}

The only significant association between induced brain activity and psychophysical pain measures implicated the right MFG within the 4.9 to $6.9 \mathrm{~Hz}$ range $(x=45, y=20, z=40)$ and involved only the change in subjective pain experienced as testing progressed through time $(r=-0.56, p=0.006$; see Figure 3$)$. This means that pain-related increases in induced theta activity within the right MFG were associated with a decrease in experienced pain. No other correlation coefficient was significant (all $\mathrm{r}$ values ( $\mathrm{rs}$ ) less than $10.44 \mathrm{l}$, all $\mathrm{p}$ values (ps) less than 0.05 ; $\mathrm{p}$ values here always include the application of Bonferroni corrections).

It is important to point out that $\mathrm{Hu}$ et al. ${ }^{15}$ recently conducted a study where they showed that post-stimulus EEG power is partly affected by the trial-to-trial variability in pre-stimulus EEG power. They also found that pre-stimulus EEG power varies following a hyperbolic function of the trial order (i.e., smaller power at the earliest trials followed by larger, relatively stable power responses thereafter). To test for the presence of trial-to-trial 
Table 1: Subjective intensity ratings and sural nerve stimulation strength $(N=23)$.

\begin{tabular}{|c|c|c|c|c|c|}
\hline & \multicolumn{4}{|c|}{ Intensity rating $[0-100]$ : mean \pm standard error } & \\
\hline & \multicolumn{3}{|c|}{ Assessments } & \multirow[b]{2}{*}{ Average } & \\
\hline & 1 & 2 & 3 & & Sural nerve stimulation $[\mathrm{mA}]:$ mean \pm standard error \\
\hline Block 1 (non painful) & $58.24 \pm 4.21$ & $53.78 \pm 4.35$ & $48.17 \pm 4.19$ & $53.40 \pm 3.70$ & $7.26 \pm 0.58$ \\
\hline Block 2 (painful): & $16.26 \pm 2.02$ & $14.74 \pm 1.83$ & $14.30 \pm 2.06$ & $15.10 \pm 1.75$ & $13.35 \pm 1.08$ \\
\hline
\end{tabular}

$\mathrm{N}=$ number; $\mathrm{mA}=$ milliampere

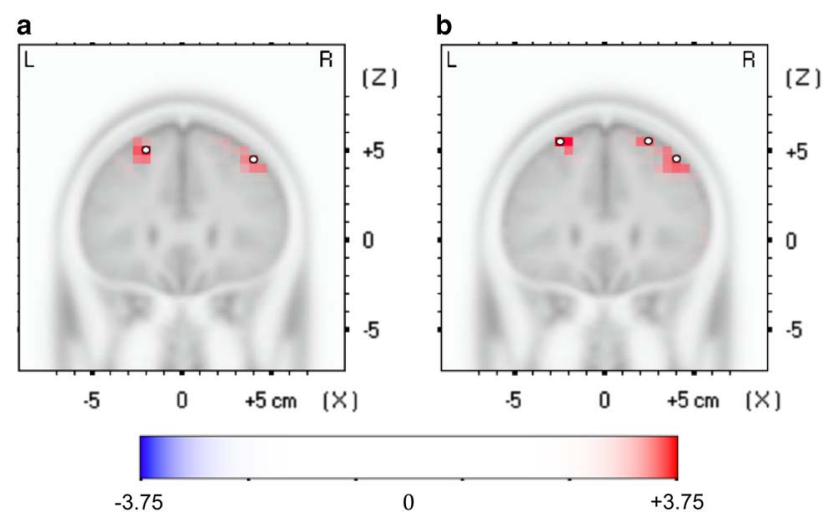

Figure 2: Coronal views $(y=30)$ showing the current density differences between painful and non-painful conditions. Significant differences were located within the left superior (1) and right middle (2) frontal gyrus in the 2.9 to $4.9 \mathrm{~Hz}$ frequency range (see a) and within the left (3) and right (4) superior frontal gyrus and in the right middle (5) frontal gyrus in the 4.9 to $6.9 \mathrm{~Hz}$ frequency range (see b). All local maxima differences (white dots) were significant. Color bar at bottom represents positive (red) and negative (blue) $t$-statistic values.

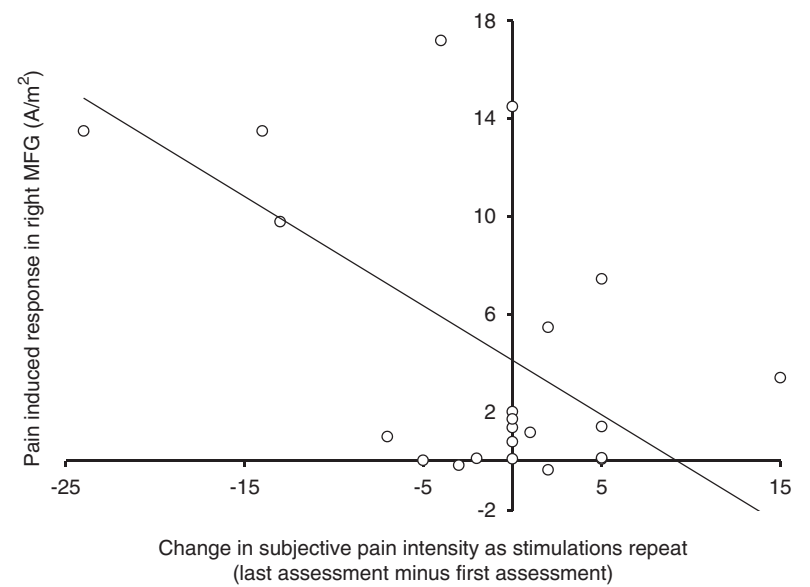

Figure 3: Induced theta brain activity obtained during the pain block and located in the right middle frontal gyrus correlated negatively with the change in subjective pain intensity (i.e., the difference between the last and first pain perception score within the pain block).

variability in pre-stimulus power and thus for the possibility of having to correct for pre-stimulus effects, we calculated theta power (via a fast-Fourier transform) within a -100 to $0 \mathrm{~ms}$ prestimulus time window for all pain trials and compared the average power obtained within the first three trials to the average power obtained in the middle three trials (trials 16, 17 and 18) and last

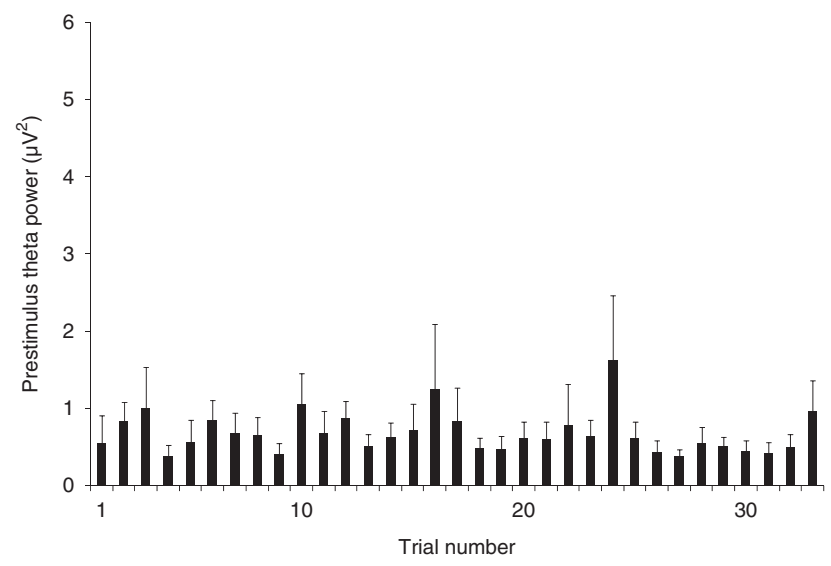

Figure 4: Average pre-stimulus theta power (between -100 to $0 \mathrm{~ms}$ ) calculated for each trial within the pain block. A significant trial effect on the modulation of pre-stimulus power values is not observed.

three trials. This was done for power values extracted from electrode $\mathrm{Oz}$, where theta power in the pain block was strongest. Results from the one-way repeated measures ANOVA conducted to test for this effect showed that trial order had no influence on pre-stimulus power $\left(\mathrm{F}_{2,44}=0.08, \mathrm{p}=0.93\right.$; see Figure 4$)$. As a result, no correction for pre-stimulus EEG power was required or carried out.

\section{Specificity of non-phase locked SEP responses}

Although our TF analyses were applied only to averageremoved single trials, this approach does not guarantee that induced (i.e., non-phase locked) components have been conclusively isolated. This is because subtracting an average response cannot completely eliminate an effect which varies from trial-totrial. Thus, to ensure that our induced effects are indeed specific (or at least not dominated by evoked effects), we re-ran our analyses using only evoked data (i.e., data obtained from single subject average responses). Since our objective here was to confirm the specificity of our induced results and not to chart the full spectrum of synchronous and asynchronous pain-evoked responses, we limited our analyses to the post-shock epoch when our induced effects were strongest (i.e., between 180 and $500 \mathrm{~ms}$ ). Visual inspection of the evoked time frequency oscillation amplitude map (Figure 5) shows greater synchronous activity for pain trials than for non-pain across multiple scalp regions and frequencies. Pain versus non-pain differences calculated specifically across frontal and posterior leads and specifically for delta/ theta frequencies were significant at frontal leads (peak activity at electrode $\mathrm{Fp} 1, \mathrm{t}_{22}=2.63, \mathrm{p}=0.01$ ) and marginal at posterior leads 


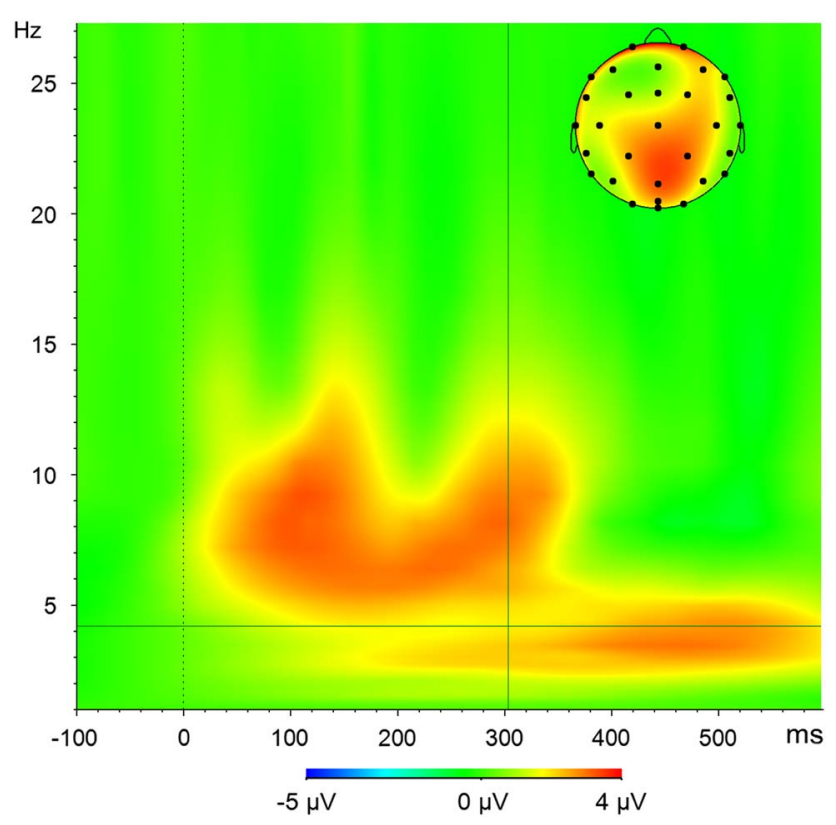

Figure 5: Time-frequency decomposition of phase locked (i.e., evoked) oscillatory activity recorded at electrode $\mathrm{Oz}$ (distribution across the entire scalp is shown in upper right corner). Time frequency data shown here represents the activity difference between pain and nonpain averages (across participants). Estimations were obtained using a continuous Morlet transform applied to both pain and non-pain averages.

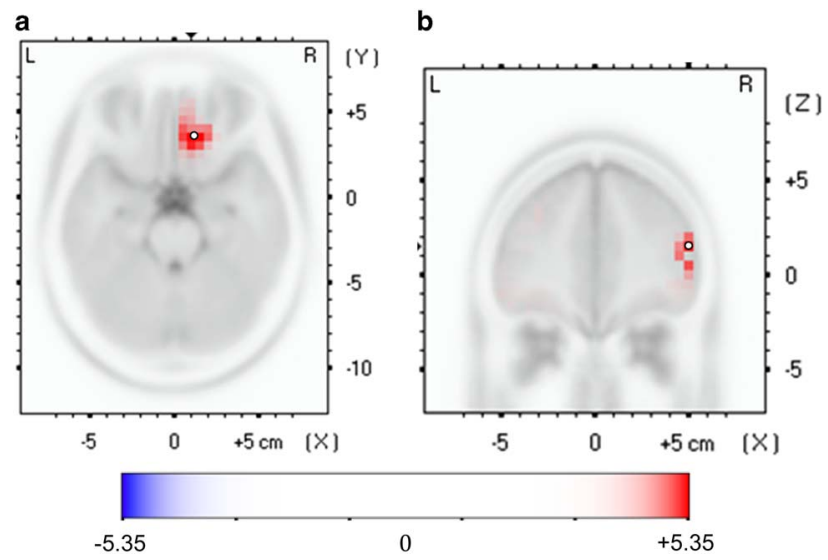

Figure 6: Current density differences between painful and non-painful conditions were significant within the right rectal gyrus in the 2.9 to $4.9 \mathrm{~Hz}$ frequency range (see a for an axial view at $z=-25$ ) and within the right inferior frontal gyrus in the 4.9 to $6.9 \mathrm{~Hz}$ frequency range (see $b$ for a coronal view at $y=40$ ). All local maxima differences (white dots) were significant. Color bar at bottom represents positive (red) and negative (blue) $t$-statistic values.

(peak activity at electrode $\mathrm{Oz}_{22}=1.76, \mathrm{p}=0.09$ ). This is pattern of activity is comparable to the pattern obtained when analyzing induced responses (see description of induced effects above). Source generators, however, were not the same as those obtained for induced responses. Within the 2.9 to $4.9 \mathrm{~Hz}$ range, generators for evoked responses were localised to the right rectal gyrus (spatial local maximum at $\mathrm{x}=10, \mathrm{y}=35, \mathrm{z}=-25 ; \mathrm{t}_{22}=5.30$, $\mathrm{p}<0.001$; see Figure 6a). Within the 4.9 to $6.9 \mathrm{~Hz}$ range, generators were localized to the right inferior frontal gyrus (spatial local maximum at $\mathrm{x}=50, \mathrm{y}=40, \mathrm{z}=15 ; \mathrm{t}_{22}=4.28, \mathrm{p}<0.05$; see Figure $6 \mathrm{~b}$ ). Neither one of these generators were associated with our psychophysical pain measures (all $\mathrm{rs}<10.421$, all ps $>0.05$; $\mathrm{p}$ values here include the application of Bonferroni corrections). This suggests that the induced effects reported earlier are not dominated (or driven) by evoked effects.

\section{Discussion}

The purpose of this study was to investigate the functional significance of non-phase locked (induced) brain responses following painful events. Results showed that following a painful electrical stimulation of the right sural nerve, a burst of delta/theta activity occurred soon after shock onset (between 180 and $500 \mathrm{~ms}$ ). This activity was source-localized to the middle and superior frontal gyri (bilaterally). Importantly, increased theta activity within the right MFG predicted decreased sensations of pain as stimulations repeated within the pain block. The MFG is an anatomical region which makes up part of the functional structure known as the dorsolateral prefrontal cortex (DLPFC). Given the association found here between DLPFC activity and subjective pain, our results suggest that the DLPFC may be associated with processes related to the attenuation of repeating painful experiences.

Results reported here agree with those of a previous positron emission tomography study conducted by Lorenz et al. ${ }^{21}$ which showed that the association between the anterior insula (a wellknown pain processing node in the human brain) and subjective pain intensity scores decrease when right DLPFC activity is high. This suggests that the DLPFC exerts active control of pain perception by modulating cortico-cortical pathways involved in the processing of nociceptive afferents. Although the Lorenz et al. paper constitutes a seminal study on the role of the prefrontal cortex in pain, the neuroimaging technique used depends on haemodynamic brain responses - which are notoriously poor at capturing rapid fluctuations in post-shock brain activity (but well suited to capture three dimensional sources). Haemodynamicbased neuroimaging data also fail to provide a direct measure of neuronal activity, let alone offer results which can distinguish between evoked and induced neuronal brain responses. In the current study, the DLPFC activity observed was induced (i.e., devoid of any contribution from the phase-locked average response) and occurred soon after shock onset, which can be instructive regarding the functional significance of this response. Variations in induced brain activity have been proposed to reflect functional changes in the parameters controlling dynamic interactions within and between brain structures (i.e. a changes in the synchrony of underlying neuronal population $\mathrm{s}^{22}$ ). This means that our rapid post-stimulus DLPFC response may represent a control process which relates to physiological connectivity in the cortex and may thus represent a measure of long-range interaction between brain structures (see Alba et al. $^{22}$ for an original formulation of this idea). This likely explains why, in our study, the DLPFC was associated with a progressive decrease in pain intensity (reflecting the exercise of control) rather than with baseline pain sensitivity (reflecting an inceptive response to pain).

In the current study, some limitations are worth mentioning. First, the association we found between pain-induced brain activity and the change in pain observed as testing advanced was based on the brain activity recorded and averaged across the entire 
pain block. As a result, we do not actually measure change in brain activity between the earliest and latest fractions of the pain block. The current testing design was simply not intended to partial out early and late block effects in the EEG response (i.e., too few trials within a block to compare brain responses to the first and last trials). Another limitation of the current study is that we do not know if the activation of the right DLPFC represents a lateralized or a right brain effect. To address this question, a testing design where both right and left sural nerve shocks are provided would be necessary. Moreover, our study cannot characterise the causal effects tied to pain-induced DLPFC responses. That is, we cannot be sure if increased DLPFC activity leads to a change in perceived pain or if a change in perceived pain leads to increased DLPFC activity. To identify causal effects related to DLPFC activity, future studies should use repetitive transcranial magnetic stimulation (rTMS), since this type of stimulation produces direct changes in underlying brain activity. Depending on rTMS frequency, it is possible to either facilitate (at high frequencies) or impede (at low frequencies) synaptic efficiency, which helps pinpoint how a given brain region relates to observed behaviour. Interestingly, a few recent pain studies have used rTMS technology to identify the role played by the DLPFC. ${ }^{23-27}$ For example, Nahmias et al. ${ }^{27}$ showed that unilateral, high frequency rTMS pulses applied to the right DLPFC produced diffuse analgesic effects in healthy human participants exposed to experimental heat and cold pain. This initial result suggests that the DLPFC may, indeed, play a causal role in reducing painful sensations.

Our findings may also have important implications regarding the comprehension of pain control processes in chronic pain patients. This is because (i) the DLPFC is considered to be part of an executive control network normally associated with pain habituation, pain coping, and pain inhibition ${ }^{28,29}$, (ii) chronic pain patients show structural, neurochemical and functional changes in the DLPFC ${ }^{19}$ and, finally, (iii) chronic pain patients fail to show adaptive coping responses (including habituation to prolonged pain) when exposed to prolonged experimental stimuli. ${ }^{30}$ Interestingly, a relatively recent study also showed that cerebral potentials recorded in response to repetitive noxious stimuli do not habituate (i.e., do not decrease in amplitude) in patients who suffer from fibromyalgia but do in healthy controls. ${ }^{31}$ This neuronal effect was further tied to reduced habituation of experienced pain. The available scientific literature, together with our results suggest that the DLPFC may serve as a promising target area in the development of neuroimaging-based risk models and/ or targeted neurostimulation approaches to treatment.

In conclusion, the current study demonstrated that a progressive decrease in perceived pain intensity was associated with a rapidly occurring burst of theta activity in the right DLPFC. Since this activity was out of phase with respect to stimulus onset, it is unlikely to be directly associated with the processing of noxious afferents but, instead, reflect the response of neuronal networks involved in the control of pain. Future studies, therefore, should investigate the connectivity between the DLPFC and well-known pain-related brain nodes, as well as investigate the role played by this structure in the development and/or maintenance of chronic pain.

\section{Disclosures}

Raphaël Dufort Rouleau has the following disclosures: Fonds de Recherche du Québec - Santé, Researcher, Research grant;
Natural Sciences and Engineering Research Council of Canada, Researcher, Research grant. Lydia Lagrandeur has the following disclosures: Fonds de Recherche du Québec - Santé, Researcher, Research grant; Natural Sciences and Engineering Research Council of Canada, Researcher, Research grant. Kathya Daigle has the following disclosures: Fonds de Recherche du Québec - Santé, Researcher, Research grant; Natural Sciences and Engineering Research Council of Canada, Researcher, Research grant. Dominique Lorrain has the following disclosures: Fonds de Recherche du Québec - Santé, Researcher, Research grant; Natural Sciences and Engineering Research Council of Canada, Researcher, Research grant. Guillaume Léonard has the following disclosures: Fonds de Recherche du Québec - Santé, Researcher, Research grant; Natural Sciences and Engineering Research Council of Canada, Researcher, Research grant. Kevin Whittingstall has the following disclosures: Fonds de Recherche du Québec - Santé, Researcher, Research grant; Natural Sciences and Engineering Research Council of Canada, Researcher, Research grant.Philippe Goffaux has the following disclosures: Fonds de Recherche du Québec - Santé, Researcher, Research grant; Natural Sciences and Engineering Research Council of Canada, Researcher, Research grant.

\section{ACKNOWLEDGEMENTS}

This work was supported by grants from the Fonds de Recherche du Québec - Santé and the Natural Sciences and Engineering Research Council of Canada. The authors report no conflict of interest concerning the materials or methods used in this study or the findings in this paper.

\section{REFERENCES}

1. Apkarian AV, Bushnell MC, Treede RD, Zubieta JK. Human brain mechanisms of pain perception and regulation in health and disease. Eur J Pain. 2005;9:463-84.

2. Borsook D, Sava S, Becerra L. The pain imaging revolution: advancing pain into the 21st century. Neuroscientist. 2010; 16:171-85.

3. Duerden EG, Albanese MC. Localization of pain-related brain activation: A meta-analysis of neuroimaging data. Hum Brain Mapp. 2013;34:109-49.

4. Peyron R, Laurent B, Garcia-Larrea L. Functional imaging of brain responses to pain. A review and meta-analysis (2000) Neurophysiol Clin. 2000;30:263-88.

5. Shao S, Shen K, Yu K, Wilder-Smith EP, Li X. Frequency-domain EEG source analysis for acute tonic cold pain perception. Clin Neurophysiol. 2012;123:2042-9.

6. Perchet C, Godinho F, Mazza S, et al. Evoked potentials to nociceptive stimuli delivered by $\mathrm{CO} 2$ or Nd:YAP lasers. Clin Neurophysiol. 2008;119:2615-22.

7. Goffaux P, Girard-Tremblay L, Marchand S, Daigle K, Whittingstall K. Individual Differences in Pain Sensitivity Vary as a Function of Precuneus Reactivity. Brain Topogr. 2014, May; 27:366-74.

8. Nir RR, Lev R, Moont R, Granovsky Y, Sprecher E, Yarnitsky D. Neurophysiology of the cortical pain network: revisiting the role of $\mathrm{S} 1$ in subjective pain perception via standardized low-resolution brain electromagnetic tomography (sLORETA). J Pain. 2008;9:1058-69.

9. Moont R, Crispel Y, Lev R, Pud D, Yarnitsky D. Temporal changes in cortical activation during conditioned pain modulation (CPM), a LORETA study. Pain. 2011;152:1469-77.

10. Pfurtscheller G, Lopes da Silva FH. Event-related EEG/MEG synchronization and desynchronization: basic principles. Clin Neurophysiol. 1994;110:1842-57.

11. David O, Kilner JM, Friston KJ. Mechanisms of evoked and induced responses in MEG/EEG. Neuroimage. 2006;31:1580-91. 
12. Mouraux A, Guerit JM, Plaghki L. Non-phase locked electroencephalogram (EEG) responses to $\mathrm{CO}_{2}$ laser skin stimulations may reflect central interactions between A partial partial differential- and C-fibre afferent volleys. Clin Neurophysiol. 2003;114:710-22.

13. Babiloni C, Babiloni F, Carducci F, et al. Human brain oscillatory activity phase-locked to painful electrical stimulations: a multichannel EEG study. Hum Brain Mapp. 2002;15:112-23.

14. Carlsson K, Andersson J, Petrovic P, Petersson KM, Ohman A, Ingvar M. Predictability modulates the affective and sensorydiscriminative neural processing of pain. Neuroimage. 2006;32: $1804-14$

15. Hu L, Xiao P, Zhang ZG, Mouraux A, Iannetti GD. Single-trial timefrequency analysis of electrocortical signals: baseline correction and beyond. Neuroimage. 2014;84:876-87.

16. Kalcher J, Pfurtscheller G. Discrimination between phase-locked and non-phase-locked event-related EEG activity. Electroencephalogr Clin Neurophysiol. 1995;94:381-4.

17. Pascual-Marqui RD, Esslen M, Kochi K, Lehmann D. Functional imaging with low-resolution brain electromagnetic tomography (LORETA): a review. Methods Find Exp Clin Pharmacol. 2002; 24(Suppl C):91-5.

18. Pascual-Marqui RD, Michel CM, Lehmann D. Low resolution electromagnetic tomography: a new method for localizing electrical activity in the brain. Int J Psychophysiol. 1994;18:49-65.

19. Schweinhardt P, Bushnell MC. Pain imaging in health and diseasehow far have we come? J Clin Invest. 2010;120:3788-97.

20. Tracey I, Mantyh PW. The cerebral signature for pain perception and its modulation. Neuron. 2007:55:377-91.

21. Lorenz J, Minoshima S, Casey KL. Keeping pain out of mind: the role of the dorsolateral prefrontal cortex in pain modulation. Brain. 2003;126:1079-91.

22. Alba A, Marroquin JL, Pena J, Harmony T, Gonzalez-Frankenberger B. Exploration of event-induced EEG phase synchronization patterns in cognitive tasks using a time-frequency-topography visualization system. J Neurosci Methods. 2007;161:166-82.

23. Borckardt JJ, Smith AR, Reeves ST, et al. Fifteen minutes of left prefrontal repetitive transcranial magnetic stimulation acutely increases thermal pain thresholds in healthy adults. Pain Res Manag. 2007;12:287-90.

24. Brighina F, De Tomasso M, Giglia F, et al. Modulation of pain perception by transcranial magnetic stimulation of left prefrontal cortex. J Headache Pain. 2011;12:185-91.

25. Krummenacher P, Candia V, Folkers G, Schedlowski M, Schonbachler G. Prefrontal cortex modulates placebo analgesia. Pain. 2010;148:368-74.

26. Martin L, Borckardt JJ, Reeves ST, et al. A Pilot Functional MRI Study of the Effects of Prefrontal rTMS on Pain Perception. Pain Med. 2013;14:999-1009.

27. Nahmias F, Debes C, de Andrade DC, Mhalla A, Bouhassira D. Diffuse analgesic effects of unilateral repetitive transcranial magnetic stimulation (rTMS) in healthy volunteers. Pain. 2009;147:224-32.

28. Buckner RL, Andrews-Hanna JR, Schacter DL. The brain's default network: anatomy, function, and relevance to disease. Ann N Y Acad Sci. 2008;1124:1-38.

29. Fox MD, Snyder AZ, Vincent JL, Corbetta M, Van E, Raichle ME. The human brain is intrinsically organized into dynamic, anticorrelated functional networks. Proc Natl Acad Sci USA. 2005;102:9673-8.

30. Smith BW, Tooley EM, Montague EQ, Robinson AE, Cosper CJ, Mullins PG. Habituation and sensitization to heat and cold pain in women with fibromyalgia and healthy controls. Pain. 2008; $140: 420-8$.

31. DeTommaso M, Federici A, Santostasi R, et al. Laserevoked potentials habituation in fibromyalgia. J Pain. 2011;12: $116-24$. 